\title{
Drenaje de pseudoquiste pancreático guiado por ecoendoscopia sin fluoroscopia: serie de casos
}

\section{Case Series of Drainage of Pancreatic Pseudocysts Guided by Echoendoscopy without Fluoroscopy}

Martín A. Gómez Zuleta, MD, ${ }^{1}$ Adán Luquez Mindiola, MD, ${ }^{2}$ Óscar Fernando Ruiz Morales, MD. ${ }^{3}$

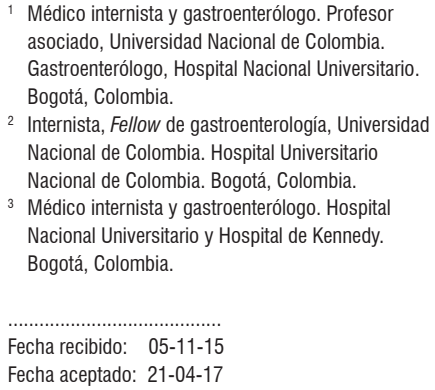

Médico internista y gastroenterólogo. Profesor asociado, Universidad Nacional de Colombia. Gastroenterólogo, Hospital Nacional Universitario. Bogotá, Colombia.

2 Internista, Fellow de gastroenterología, Universidad Nacional de Colombia. Hospital Universitario Nacional de Colombia. Bogotá, Colombia.

${ }^{3}$ Médico internista y gastroenterólogo. Hospital Nacional Universitario y Hospital de Kennedy. Bogotá, Colombia.

Fecha recibido: $\quad 05-11-15$ Fecha aceptado: 21-04-17

\begin{abstract}
Resumen
Introducción: los pseudoquistes pancreáticos pueden ser drenados por métodos quirúrgicos, laparoscópicos, percutáneos y endoscópicos. Este último es el más aceptado en la actualidad dado que es más simple y genera menos morbimortalidad; sin embargo, siempre se ha descrito asociado con el uso de la fluoroscopia, lo cual le suma complejidad. En este trabajo presentamos nuestra técnica de drenaje sin fluoroscopia guiado por ecoendoscopia. Materiales y métodos: el objetivo de este trabajo es describir una técnica de drenaje de pseudoquistes pancreáticos sin el uso de fluoroscopia y con la guía del ecoendoscopio. Se reporta una serie de casos de 10 pacientes a quienes se les realizó drenaje transgástrico, en los que se describen la técnica, complicaciones y resultados en el seguimiento. Resultados: se incluyeron 10 pacientes consecutivos, 5 pacientes eran mujeres y 5 hombres. El $90 \%$ de los casos comprometía el cuerpo del páncreas. Se implantó exitosamente 1 stent (metálico) transmural (cistogastrostomía) solo con guía ecoendoscópica en 9 pacientes; en 1 paciente no se logró liberar el stent, por lo cual se realizó el drenaje por aspiración. Los 9 pacientes a quienes se les implantó stent no presentaron recidiva; la paciente a quien se realizó drenaje por aspiración presentó recidiva a nivel del cuerpo de páncreas. La principal complicación fue la migración del stent hacia la cavidad del pseudoquiste en 1 paciente, el cual requirió segundo tiempo endoscópico para retirarlo. Conclusiones: el drenaje transmural del pseudoquiste pancreático con implante de stent es una técnica mínimamente invasiva, segura y efectiva para el tratamiento del pseudoquiste pancreático.
\end{abstract}

Palabras clave

Pancreatitis, pseudoquiste, ecoendoscopia, drenaje.

\begin{abstract}
Introduction: Pancreatic pseudocysts can be drained by surgical, laparoscopic, percutaneous, and endoscopic methods. Endoscopic methods have become the most widely accepted nowadays since they are simpler and generate less morbidity and mortality. They have always been associated with the use of fluoroscopy, which adds complexity. This study presents our drainage technique which is guided by echoendoscopy rather than fluoroscopy. Materials and methods: The objective of this study is to describe a technique for drainage of pancreatic pseudocysts which does not use fluoroscopy to guide the endoscope. Instead, echoendoscopy guides the instrument. We report a case series of 10 patients who underwent transgastric drainage and describe the technique, complications and results during follow-up. Results: Ten consecutive patients, five women and five men, were included in this study. Ninety percent were cases in which the body of the pancreas had been compromised. Cystogastrostomy was successfully performed in nine patients. It consisted of implantation of a transmural metallic stent under single endoscopic guidance. In one patient the stent was not released and aspiration drainage was performed. The nine patients who had stents implanted have presented no recurrences, but the patient who underwent aspiration drainage presented recurrence in the body of the pancreas. The main complication was migration of the stent into the pseudocyst cavity which occurred in one patient and which required a second endoscopic procedure to remove the stent. Conclusions: Transmural drainage of pancreatic pseudocysts through placement of stents is a safe, effective and minimally invasive technique for the treatment of pancreatic pseudocysts.
\end{abstract}

\section{Keywords}

Pancreatitis, pseudocysts, echoendoscopy, drainage. 


\section{INTRODUCCIÓN}

El pseudoquiste pancreático se define como una colección líquida en el tejido peripancreático o intrapancreático que está rodeada por una pared bien definida y contiene principalmente material no sólido (1). El pseudoquiste pancreático es una complicación local debido a la ruptura del conducto pancreático en pancreatitis aguda o crónica, trauma u obstrucción del conducto pancreático. Este daño del conducto conlleva a la acumulación del jugo pancreático, el cual es encerrado por una pared no epitelizada en un período de 4 a 6 semanas y se forma el pseudoquiste (2).

La mayoría de los pseudoquistes pancreáticos son asintomáticos, los síntomas ocurren durante el curso clínico de la enfermedad asociado con complicaciones y principalmente se presenta con dolor abdominal, fiebre, ictericia, saciedad precoz, pérdida de peso, sangrado y emesis (3).

Las indicaciones de drenaje de estos pseudoquistes son: dolor persistente, obstrucción gástrica o duodenal, obstrucción biliar, ascitis, derrame pleural, aumento de tamaño progresivo durante el seguimiento imagenológico, signos de infección o sangrado, la posibilidad de malignidad quística pancreática y el tamaño $>6 \mathrm{~cm}$; pero con respecto al tamaño hay resultados divergentes dado que, si están completamente asintomáticos, no ameritarían manejo (4).

Los pseudoquistes pancreáticos pueden ser drenados por métodos quirúrgicos, laparoscópicos, percutáneos y endoscópicos (5-7). Tradicionalmente, el manejo quirúrgico era el tratamiento de elección, pero por el desarrollo de las técnicas endoscópicas en los últimos años el manejo ha evolucionado a abordajes mínimamente invasivos (8). Los pseudoquistes pancreáticos pueden ser drenados endoscópicamente por un abordaje transpapilar o transmural según esté comunicado con el conducto pancreático principal, y algunas veces puede ser necesaria la combinación de ambos métodos $(2,8)$. El drenaje transmural se logra insertando un stent entre el pseudoquiste pancreático y la luz gástrica (cistogastrostomía) o duodenal (cistoduodenostomía), se puede realizar por vía endoscópica directa como un procedimiento semiciego si hay un abultamiento gástrico o duodenal producido por el quiste o bajo visión ecoendoscópica con tasas significativamente más altas de éxito $(4,9-11)$. El drenaje transmural guiado por ecoendoscopia se puede realizar bajo visión ultrasonográfica sola o combinado con fluoroscopia; la ecoendoscopia es necesaria para guiar la aguja dentro de la cavidad quística y la fluoroscopia es necesaria para confirmar el acceso dentro de la cavidad quística por inyección de medio de contraste, para el enrollamiento de la guía hidrofílica en la cavidad y mientras se libera el stent. La técnica descrita consiste en ubicar el pseudoquiste por ecoendoscopia, puncionar con una aguja número 19ga de ecoendoscopia, retirar el estilete y por la aguja se aplica medio de contraste, lo que permite identificar completamente el quiste en la fluoroscopia; ulteriormente se introduce una guía hidrofílica de 4,5 $\mathrm{mm}$ y se deja en la cavidad del quiste dándole varias vueltas para asegurar el acceso, se retira la aguja de punción y sobre la guía se pasa un dilatador biliar de 7 French (Fr) con pistola neumática, se insufla el balón y se dilata el trayecto que comunica al quiste con la pared gástrica para pasar el stent, que puede ser plástico doble cola de cerdo o metálico autoexpandible (12-15). El stent se retira a las 6 u 8 semanas una vez la tomografía axial computarizada (TAC) de abdomen control verifique que no hay colección residual (13).

En esta serie de casos se reporta la experiencia de nuestro grupo con el drenaje de pseudoquiste pancreático utilizando una nueva técnica: guiado por ultrasonografía endoscópica sin requerimiento de fluoroscopia y sin dilatación de la pared gástrica.

\section{MATERIALES Y MÉTODOS}

Este trabajo presenta una serie consecutiva de pacientes entre diciembre de 2013 y julio de 2015 con diagnóstico de pseudoquiste pancreático sintomático.

Los procedimientos fueron realizados en la sala de gastroenterología del Hospital El Tunal bajo sedación guiada por anestesiólogo con una combinación de propofol más remifentanilo. Todos los drenajes fueron transmurales guiados por ecoendoscopia con un equipo marca Pentax, un endosonógrafo lineal y realizado por un endoscopista experimentado en procedimientos intervencionistas. En todos los casos se implantaron stents metálicos autoexpandibles parcial o totalmente recubiertos y en algunos casos se colocó dentro del stent metálico un stent doble cola de cerdo para prevenir la migración.

Se realizó control con TAC de abdomen con contraste 6 semanas después del procedimiento para evaluar la evolución del pseudoquiste pancreático. Una vez resuelto el pseudoquiste, se realizó endoscopia digestiva alta para retirar el stent transmural.

\section{Descripción de la técnica}

La técnica que utilizamos y la cual evaluamos en este estudio fue la siguiente: una vez ubicado el pseudoquiste con el ecoendoscopio lineal y a la frecuencia de $7,5 \mathrm{MHz}$, se aplica el Doppler en la pared que separa el quiste del estómago para descartar vasos importantes. Luego, con aguja de punción número 19ga (expect-Boston) se punciona el quiste y se fija el seguro que permite desplazar la aguja, se aspira el líquido y se envía una muestra para tinción de Gram y cultivo si es necesario. El estilete se retira y se avanza la guía dando unas 3 a 4 vueltas dentro del quiste, se libera 
el seguro que permite desplazar la camisa de la aguja y se repite la punción, pero solo con la camisa (dejando fijo el seguro que permite desplazar la aguja), lo que permite ampliar el orificio que comunica el quiste con la luz y se retira la aguja, y una vez retirada la aguja se deja la guía y se coloca el tapón de caucho en el canal de trabajo para evitar que el aire se escape, lo que dificulta el procedimiento. Sobre la guía se monta el stent metálico autoexpandible (10 $\mathrm{mm}$ x $60 \mathrm{~mm}$ o $10 \mathrm{~mm}$ x $80 \mathrm{~mm}$ ), luego se libera observando primero cómo se abre el extremo distal en la ecografía y, después, en la imagen endoscópica se observa cómo se libera el extremo proximal.

\section{RESULTADOS}

Se incluyeron 10 pacientes con diagnóstico de pseudoquiste pancreático (Figura 1) a quienes se les realizó drenaje transmural por ecoendoscopia. Las variables demográficas principales se presentan en la Tabla 1.

5 pacientes eran mujeres (50\%). El rango de edad de presentación estaba entre 11 y 68 años de edad. El diámetro promedio fue $10,6 \mathrm{~cm}(5-22 \mathrm{~cm})$. El $90 \%$ de los casos comprometía el cuerpo de páncreas. Se logró implantar exitosamente un stent transmural (cistogastrostomía) en 9 pacientes (90\%) (Figura 2), en 6 pacientes se colocó solo el stent metálico autoexpandible parcial o totalmente recubierto y en 3 pacientes se colocó el stent metálico y en su interior un stent doble cola de cerdo de $10 \mathrm{Fr}$, en 1 paciente se realizó el drenaje por aspiración con resolución del 95\%

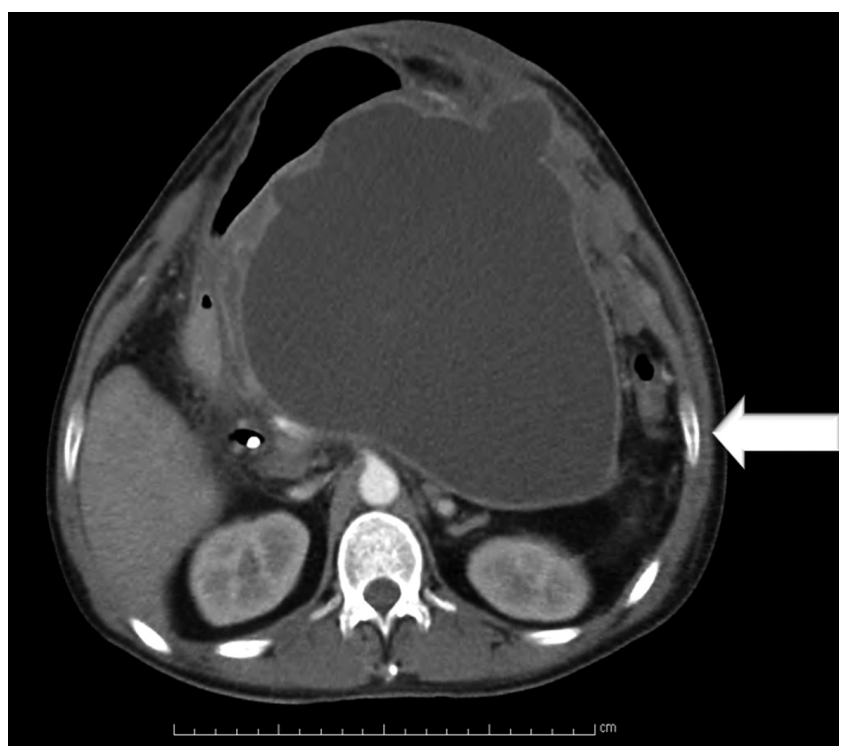

Figura 1. TAC de abdomen con contraste. Pseudoquiste pancreático. La flecha señala el pseudoquiste pancreático.

del tamaño de la lesión dado que el pseudoquiste fue de $5 \mathrm{~cm}$. Un paciente presentó durante el procedimiento liberación del stent metálico dentro de la cavidad del pseudoquiste, requirió la colocación de otro stent metálico y en un segundo tiempo endoscópico, 48 horas después, se retiró el stent entrando a la cavidad con un endoscopio pediátrico y luego se reposicionó el stent migrado.

Tabla 1. Variables demográficas de los pacientes

\begin{tabular}{|c|c|c|c|c|c|c|c|c|}
\hline Paciente & $\begin{array}{l}\text { Género } \\
\text { (M/F) }\end{array}$ & $\begin{array}{l}\text { Edad } \\
\text { (años) }\end{array}$ & Indicación & Localización & $\begin{array}{l}\text { Tamaño } \\
(\mathrm{cm})\end{array}$ & Hallazgo & Stent & Complicación \\
\hline 1 & M & 59 & PP & Cuerpo & 12 & NPA & MTR $10 \times 60 \mathrm{~mm}$ & No \\
\hline 2 & M & 49 & PP & Cuerpo & 22 & PP & MTR $10 \times 60 \mathrm{~mm}$ & No \\
\hline 3 & $\mathrm{~F}$ & 26 & PP & Cola & 10 & PP & MTR $10 \times 80 \mathrm{~mm}$ & No \\
\hline 4 & $\mathrm{~F}$ & 61 & PP & Cuerpo & 6 & PP & $\begin{array}{l}\text { MTR } 10 \times 60 \mathrm{~mm} \text { y DCC } 10 \\
\operatorname{Fr} \times 10 \mathrm{~cm}\end{array}$ & No \\
\hline 5 & M & 52 & PP & Cuerpo & 20 & PP & MTR $10 \times 80 \mathrm{~mm}$ & No \\
\hline 6 & $\mathrm{~F}$ & 60 & PP & Cuerpo y cola & 6 & PP & $\begin{array}{l}\text { MTR } 10 \times 60 \mathrm{~mm} \text { y DCC } 10 \\
\mathrm{Fr} \times 10 \mathrm{~cm}\end{array}$ & No \\
\hline 7 & M & 62 & PP & Cuerpo & 8 & PP & $\begin{array}{l}\text { MTR \#2 } \\
10 \times 60 \mathrm{~mm}\end{array}$ & $\begin{array}{l}\text { Liberación fallida de } 1 \\
\text { stent (intrapseudoquiste) }\end{array}$ \\
\hline 8 & $F$ & 51 & PP & Cuerpo & 5 & PP & Aspiración & No \\
\hline 9 & M & 11 & PP & Cuerpo & 10 & PP & $\begin{array}{l}\text { MTR } 10 \times 60 \mathrm{~mm} \text { y DCC } \\
10 \mathrm{Fr} \times 10 \mathrm{~cm}\end{array}$ & No \\
\hline 10 & $\mathrm{~F}$ & 68 & PP & Cuerpo & 7 & NPA & MPR $10 \times 60 \mathrm{~mm}$ & No \\
\hline
\end{tabular}

DCC: doble cola de cerdo; F: femenino; M: masculino; MPR: metálico parcialmente recubierto; MTR: metálico totalmente recubierto; NPA: necrosis pancreática amurallada; PP: pseudoquiste pancreático. 


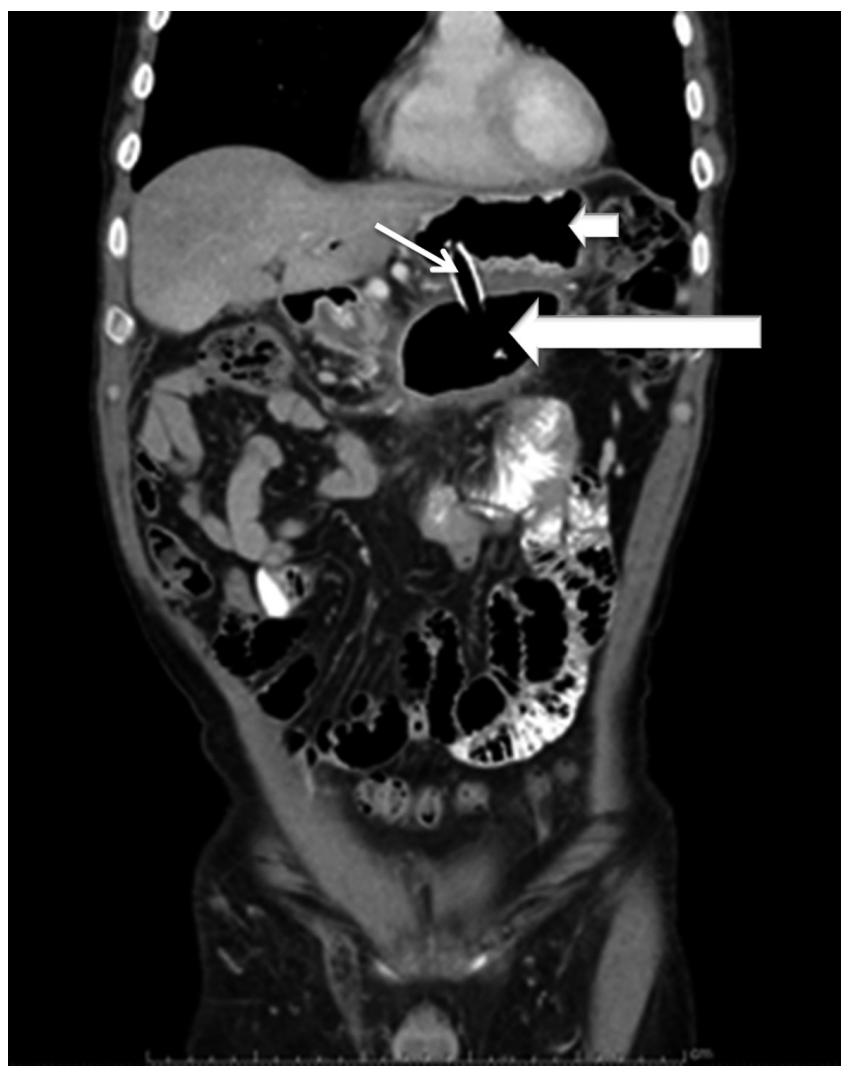

Figura 2. TAC de abdomen con contraste, control 1 semana (seguimiento al paciente de la Figura 1). Pseudoquiste pancreático con drenaje transmural. La flecha corta señala la cavidad gástrica, la flecha delgada señala el stent comunicando la cavidad gástrica y pancreática y la flecha larga señala la cavidad del pseudoquiste pancreático.

Según los hallazgos ecoendoscópicos, el $80 \%$ de las lesiones correspondía a pseudoquiste pancreático y el $20 \%$ a colección líquida con necrosis pancreática amurallada (walled-off). No hubo muertes asociadas con el procedimiento. Se realizó control tomográfico abdominal 6 semanas después del procedimiento (Figura 3 ) en el que se evidenció una resolución completa del pseudoquiste en el 90\% de los casos sin complicaciones. Todos los pacientes con cistogastrostomía resolvieron completamente la lesión, la paciente a quien se realizó drenaje por aspiración presentó recidiva del pseudoquiste a nivel del cuerpo del páncreas a las 4 semanas.

\section{DISCUSIÓN}

El drenaje transmural (cistogastrostomía) de los pseudoquistes pancreáticos es un procedimiento endoscópico mínimamente invasivo. Con el aumento de la experiencia en ecoendoscopia terapéutica, el drenaje del pseudoquiste pancreático por este medio está aumentando y es la primera línea de tratamiento para las colecciones líquidas pancreáticas sintomáticas y reemplaza el abordaje quirúrgico y percutáneo (16).

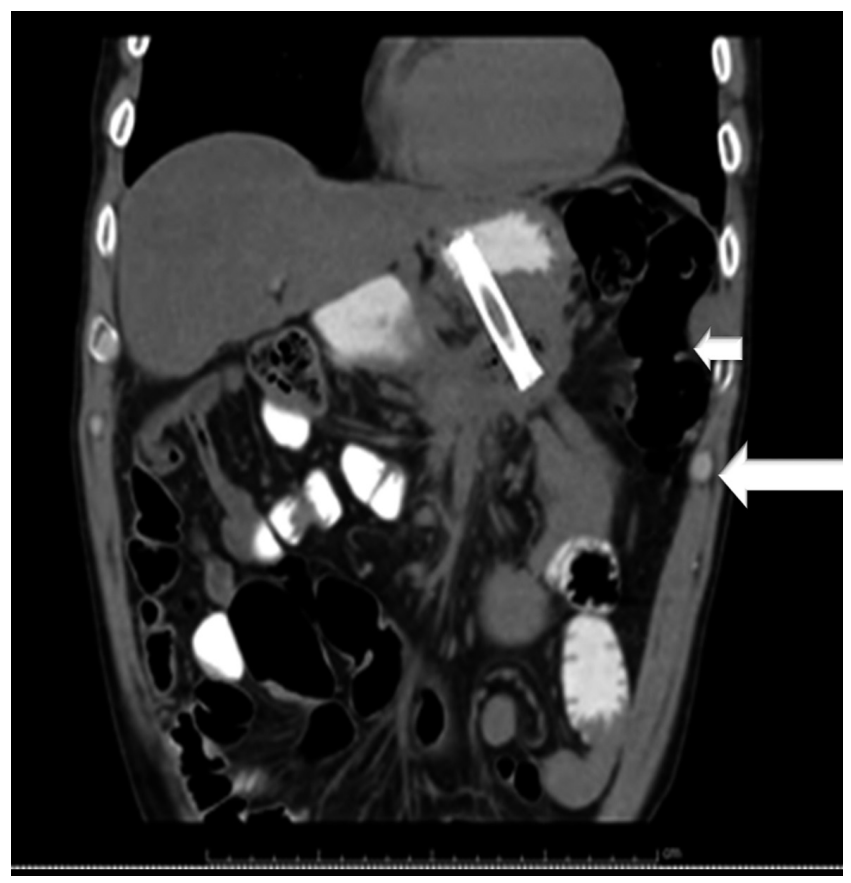

Figura 3. TAC de abdomen con contraste, control a las 6 semanas (seguimiento paciente de la Figura 1). Pseudoquiste pancreático con drenaje transmural. La flecha corta señala la cavidad gástrica en la que se observa medio de contraste y la flecha larga señala el pseudoquiste pancreático drenado.

En nuestra serie la tasa de éxito, estudio y recurrencia es similar a la encontrada en estudios publicados previamente que requiere solo un procedimiento endoscópico en el $90 \%$ de los casos (17-19).

Las complicaciones más frecuentemente asociadas con el procedimiento son sangrado, infección, neumoperitoneo, embolismo aéreo y perforación $(17,20-22)$, las cuales no se presentaron en nuestra serie. La complicación presentada en nuestra serie fue la liberación intralesión de un stent metálico; sin embargo, no se necesitó procedimiento quirúrgico para extraerlo y se presentó por no coordinar la liberación ecográfica con la visión endoscópica.

Las ventajas de la técnica descrita por nuestro grupo fueron:

1. No necesitar el uso de fluoroscopia, lo que no solo ahorra costos sino que también evita la exposición de radiaciones peligrosas para el paciente y el grupo médico.

2. No necesitar el uso de balón de dilatación para ampliar el tracto entre pared y el quiste, lo que ahorra tiempo del procedimiento y dinero, ya que el costo del accesorio no es despreciable.

Las desventajas serían:

1. Necesidad de entrenamiento y experiencia en ecoendoscopia para identificar en el ecógrafo los accesorios y cómo va abriendo el stent metálico. 
2. Requerir una coordinación mayor con el equipo para liberar los accesorios, ya que no se cuenta con la guía fluoroscópica.

En la literatura es discutible qué accesorio es mejor para drenar el pseudoquiste. Algunos grupos, como el de Varadarajulu y colaboradores, prefieren solo utilizar 2 stents plásticos doble cola de cerdo; este método ha mostrado buenos resultados y bajas tasas de migración porque las colas del stent se enrollan en el quiste. Otros grupos, como el nuestro, prefieren utilizar stent metálico porque, aunque es más costoso, es más fácil de colocar y al permitir un diámetro de drenaje mayor $(10 \mathrm{~mm}$ del stent metálico contra $3 \mathrm{~mm}$ del stent plástico doble cola de cerdo) permite un mejor y más rápido drenaje de la colección, aunque tiene más riesgo de migración, lo que se podría evitar con la técnica de colocar dentro del stent metálico un stent doble cola de cerdo $(5,10,23)$.

Esta serie, al igual que todas las que han usado stent metálico, muestra una tasa de resolución excelente (>90\%) con ninguna recidiva en el seguimiento a 6 meses y menos eventos adversos, por lo que consideramos que es el método actual de elección para drenaje.

En la literatura hay diferentes recomendaciones con respecto al momento ideal para el retiro del stent; en nuestra serie se retiraron los stent transmurales a las 6 semanas; sin embargo, estudios recientes proponen el retiro temprano a las 3 semanas, lo cual aún no ha sido evaluado por nuestro grupo y consideramos prematuro debido a que la fistula aún no ha madurado y hay alto riesgo de recidiva (24).

Muchos autores han utilizado control fluoroscópico para optimizar el acceso al pseudoquiste, aunque en la presente serie no se utiliza guía fluoroscópica por la eficacia, seguridad y beneficios referidos del drenaje guiado por ecoendoscopia (25).

En conclusión, el drenaje transmural guiado por ecoendoscopia sin fluoroscopia es una técnica mínimamente invasiva, eficaz y segura para el tratamiento del pseudoquiste pancreático sintomático, como lo demuestra esta serie; pero dado su pequeño tamaño se requieren estudios más grandes para confirmar estos resultados.

\section{REFERENCIAS}

1. Banks PA, Bollen TL, Dervenis C, et al. Classification of acute pancreatitis--2012: revision of the Atlanta classification and definitions by international consensus. Gut. 2013;62(1):10211. Doi: https://doi.org/10.1136/gutjnl-2012-302779

2. Varadarajulu S, Rana SS, Bhasin DK. Endoscopic therapy for pancreatic duct leaks and disruptions. Gastrointest Endosc Clin N Am. 2013;23(4):863-92. Doi: https://doi. org/10.1016/j.giec.2013.06.008
3. Gumaste VV, Aron J. Pseudocyst management: endoscopic drainage and other emerging techniques. J Clin Gastroenterol 2010;44(5):326-31. Doi: https://doi. org/10.1097/mcg.0b013e3181cd9d2f

4. Samuelson AL, Shah RJ. Endoscopic management of pancreatic pseudocysts. Gastroenterol Clin N Am. 2012;41(1):4762. Doi: https://doi.org/10.1016/j.gtc.2011.12.007

5. Varadarajulu S, Bang JY, Sutton BS, et al. Equal efficacy of endoscopic and surgical cystogastrostomy for pancreatic pseudocyst drainage in a randomized trial. Gastroenterology. 2013;145(3):583-90.e1. Doi: https://doi.org/10.1053/j. gastro.2013.05.046

6. Johnson MD, Walsh RM, Henderson JM, et al. Surgical versus nonsurgical management of pancreatic pseudocysts. J Clin Gastroenterol. 2009;43(6):586-90. Doi: https://doi. org/10.1097/MCG.0b013e31817440be

7. Baron TH. Endoscopic drainage of pancreatic pseudocysts. J Gastrointest Surg. 2008;12(2):369-372. Doi: https://doi. org/10.1007/s11605-007-0334-5

8. Zerem E, Hauser G, Loga-Zec S, et al. Minimally invasive treatment of pancreatic pseudocysts. World J Gastroenterol. 2015;21(22):6850-60.

9. Nasr JY, Chennat J. Endoscopic ultrasonography-guided transmural drainage of pseudocysts. Tech Gastrointest Endosc.2012;14(4):195-8.Doi: https://doi.org/10.1016/j. tgie.2012.07.001

10. Varadarajulu S, Christein JD, Tamhane A, et al. Prospective randomized trial comparing EUS and EGD for transmural drainage of pancreatic pseudocysts (with videos). Gastrointest Endosc. 2008;68(6):1102-11. Doi: https:// doi.org/10.1016/j.gie.2008.04.028

11. Park DH, Lee SS, Moon SH, et al. Endoscopic ultrasound-guided versus conventional transmural drainage for pancreatic pseudocysts: a prospective randomized trial. Endoscopy. 2009;41(10):842-8. Doi: https://doi. org/10.1055/s-0029-1215133

12. Fabbri C, Luigiano C, Maimone A, et al. Endoscopic ultrasound-guided drainage of pancreatic fluid collections. World J Gastrointest Endosc. 2012;4(11):479-88. Doi: https://doi.org/10.4253/wjge.v4.i11.479

13. Kawakami H, Itoi T, Sakamoto N. Endoscopic ultrasoundguided transluminal drainage for peripancreatic fluid collections: where are we now? Gut Liver. 2014;8(4):341-55. Doi: https://doi.org/10.5009/gnl.2014.8.4.341

14. Holt BA, Varadarajulu S. The endoscopic management of pancreatic pseudocysts (with videos). Gastrointest Endosc. 2015;81(4):804-12. Doi: https://doi.org/10.1016/j. gie.2014.12.026

15. Rana SS, Bhasin DK. Nonfluoroscopic endoscopic ultrasound-guided transmural drainage of pseudocysts: a pictorial technical review. Endosc Ultrasound. 2015;4(2):92-7. Doi: https://doi.org/10.4103/2303-9027.156719

16. Singhal S, Rotman SR, Gaidhane M, et al. Pancreatic fluid collection drainage by endoscopic ultrasound: an update. Clin Endosc. 2013;46(5):506-14. Doi: https://doi. org/10.5946/ce.2013.46.5.506 
17. Lopes CV, Pesenti C, Bories E, et al. Endoscopic ultrasound-guided endoscopic transmural drainage of pancreatic pseudocysts. Arq Gastroenterol. 2008;45(1):17-21. Doi: https://doi.org/10.1590/S0004-28032008000100004

18. Dohmoto M, Akiyama K, Lioka Y. Endoscopic and endosonographic management of pancreatic pseudocyst: a longterm follow-up. Rev Gastroenterol Peru. 2003;23(4):269-75.

19. Vosoghi M, Sial S, Garrett B, et al. EUS-guided pancreatic pseudocyst drainage: review and experience at HarborUCLA Medical Center. MedGenMed. 2002;4(3):2.

20. Lopes CV, Pesenti C, Bories E, et al. Endoscopic ultrasoundguided endoscopic transmural drainage of pancreatic pseudocysts and abscesses. Scand J Gastroenterol. 2007;42(4):5249. Doi: https://doi.org/10.1080/00365520601065093

21. Ardengh JC, Coelho DE, Coelho JF, et al. Single-step EUSguided endoscopic treatment for sterile pancreatic collections: a single-center experience. Dig Dis. 2008;26(4):3706. Doi: https://doi.org/10.1159/000177024

22. Jow AJ, Wan D. Complication of cardiac air embolism during ERCP and EUS-assisted cyst-gastrostomy for pan- creatic pseudocyst. Gastrointest Endosc. 2012;75(1):220-1. Doi: https://doi.org/10.1016/j.gie.2011.01.047

23. Sharaiha RZ, DeFilipis EM, Kedia P, et al. Metal versus plastic for pancreatic pseudocyst drainage: clinical outcome and success. Gastrointest Endosc. 2015;82(5):822-7. Doi: https://doi.org/10.1016/j.gie.2015.02.035

24. Dhir V, Bin Teoh AY, Bapat M, et al. EUS-guided pseudocyst drainage: prospective evaluation of early removal of fully covered self-expandable metal stent with pancreatic ductal stenting in selected patients. Gastrointest Endosc. 2015;82(4):650-7; quiz 718.e1-5. Doi: https://doi. org/10.1016/j.gie.2015.01.061 https://doi.org/10.1016/j. gie.2015.03.025

25. Seicean A, Stan-luga R, Badea R, et al. The safety of endoscopic ultrasonography-guided drainage of pancreatic fluid collections without fluoroscopic control: a single tertiary center experience. J Gastrointestin Liver Dis. 2011;20(1):39-45. 